\title{
An evaluation of alternative methods for constructing phylogenies from whole genome sequence data: a case study with Salmonella
}

Comparative genomics based on whole genome sequencing (WGS) is increasingly being applied to investigate questions within evolutionary and molecular biology, as well as questions concerning public health (e.g., pathogen outbreaks). Given the impact that conclusions derived from such analyses may have, we have evaluated the robustness of clustering individuals based on WGS data to three key factors: 1) next-generation sequencing (NGS) platform (HiSeq, MiSeq, lonTorrent, 454, and SOLiD), 2) algorithms used to construct a SNP (single nucleotide polymorphism) matrix (reference-based and reference-free), and 3) phylogenetic inference method (FastTreeMP, GARLI, and RAxML). We carried out these analyses on 194 whole genome sequences representing 107 unique Salmonella enterica subsp. enterica ser. Montevideo strains. Reference-based approaches for identifying SNPs produced trees that were significantly more similar to one another than those produced under the reference-free approach. Topologies inferred using a core matrix (i.e., no missing data) were significantly more discordant than those inferred using a non-core matrix that allows for some missing data. However, allowing for too much missing data likely results in a high false discovery rate of SNPs. When analyzing the same SNP matrix, we observed that the more thorough inference methods implemented in GARLI and RAxML produced more similar topologies than FastTreeMP. Our results also confirm that reproducibility varies among NGS platforms where the MiSeq had the lowest number of pairwise differences among replicate runs. Our investigation into the robustness of clustering patterns illustrates the importance of carefully considering how data from different platforms are combined and analyzed. We found clear differences in the topologies inferred, and certain methods performed significantly better 
than others for discriminating between the highly clonal organisms investigated here. The methods supported by our results represent a preliminary set of guidelines and a step towards developing validated standards for clustering based on whole genome sequence data. 
1 An evaluation of alternative methods for constructing phylogenies

2 from whole genome sequence data: A case study with Salmonella

4 Authors and affiliations:

5 James B. Pettengill ${ }^{1}$

6 Yan Lou ${ }^{1}$

7 Steven Davis

8 Yi Chen $^{1}$

James.Pettengill@fda.hhs.gov

9 Narjol Gonzalez-Escalona ${ }^{1} \quad$ Narjol.Gonzalez-Escalona@fda.hhs.gov

10 Andrea Ottesen ${ }^{1}$

Andrea.Ottesen@fda.hhs.gov

11 Hugh Rand ${ }^{1}$ Hugh.Rand@fda.hhs.gov

12 Marc W. Allard ${ }^{1}$

13 Errol Strain ${ }^{1,2}$

Marc.Allard@fda.hhs.gov

Errol.Strain@fda.hhs.gov

$14{ }^{1}$ Center for Food Safety \& Applied Nutrition, U.S. Food \& Drug Administration,

155100 Paint Branch Parkway, College Park, MD USA

$16{ }^{2}$ Corresponding author

17 Keywords: congruence, next generation sequencing, outbreak, phylogenetics, 18 Salmonella, single nucleotide polymorphism 


\section{Background}

20 The increasing availability and decreasing costs of next-generation

21 sequencing (NGS) has made it possible to apply this technique to investigate

22 non-model organisms and myriad questions within evolutionary and

23 molecular biology. Next-generation sequencing as a means to acquire whole

24 genome sequences (WGS) has also revolutionized applied research, where it

25 is now, for example, being employed to investigate issues related to public

26 health such as pathogenic outbreaks (Lienau et al. 2011; Parkhill \& Wren

27 2011; Roetzer et al. 2013; Underwood et al. 2013). In this situation, the vastly

28 increased information content in WGS data provides a genome perspective of

29 differences among isolates, which can be used to identify gene specific

30 differences associated with virulence and pathogenicity. The information

31 content within WGS data also provides superior discriminatory power with

32 which the evolutionary relationships among highly clonal strains can be

33 inferred. This phylogeny can then be used to identify clinical cases properly

34 belonging to outbreaks and establish links between clinical and

35 environmental isolates.

36 Outbreaks have been studied with NGS both retrospectively, as a means to

37 better understand a past outbreak, and during an outbreak, to aid in real

38 time decision-making. A well known example illustrating the utility of NGS to

39 investigate a food-borne disease outbreak is that of the virulent Shiga toxin

40 (Stx)-producing Escherichia coli O104: $\mathrm{H} 4$ associated with contaminated

41 sprouts in Germany; NGS data has been used to better understand

42 differences in virulence (Bielaszewska et al. 2011) and evolutionary

43 relationships (Mellmann et al. 2011) among outbreak and non-outbreak

44 isolates. A number of recent cases of salmonellosis also have been

45 investigated by clustering isolates based on SNP (single nucleotide

46 polymorphism) differences identified by WGS data. For example, two studies,

47 which used data produced on different NGS platforms and using different

48 bioinformatic methods, unequivocally identified the source of a multistate

49 outbreak of Salmonella enterica subsp. enterica serovar Montevideo

50 associated with spiced-meat (Allard et al. 2012; den Bakker et al. 2011). With

51 regards to human diseases, phylogenetic reconstruction based on WGS data

52 proved useful in the investigation and determination of the source of Vibrio

53 cholerae associated with an outbreak that occurred in Haiti in 2010 (Chin et

54 al. 2011; Hendriksen et al. 2011; Keim et al. 2011).

55 Recent studies in the hospital setting illustrate how NGS can contribute to the 56 real-time management of disease outbreaks. Koser et al. (2012) utilized WGS 
57 data to rapidly investigate an outbreak of methicillin-resistant

58 Staphylococcus aureus (MRSA) within a neonatal intensive care unit. Such an

59 approach was able to distinguish among isolates that belonged to a single

60 lineage, which was likely impossible using traditional typing methods. As a

61 result, NGS data was shown to rapidly (i.e., within a timeframe that could

62 influence patient health) provide clinically important data (Koser et al. 2012).

63 Health officials investigating a hospital outbreak of Klebsiella pneumoniae,

64 found that using whole-genome sequencing yielded "actionable insights" by

65 discerning among alternative transmission routes and, thus, containment of

66 the infection (Snitkin et al. 2012).

67 The recent publication dates of the studies discussed above illustrate just

68 how new the use of NGS data within public health is. Given the potential

69 impact of public health decisions based on NGS data, the robustness of NGS-

70 based results must be evaluated. Three factors that may differ within or

71 among studies are 1) the NGS platform on which the sequences were

72 generated, 2) the method used to detect variant sites and create a SNP

73 matrix, and 3) the phylogenetic inference method used to cluster samples.

74 With regards to NGS platform, it is well documented that performance (e.g.,

75 error rates and error structure) differs among them (Harismendy et al. 2009;

76 Loman et al. 2012; Mardis 2013; Shendure \& Ji 2008). Although some may

77 argue this is not a significant issue as certain platforms are no longer

78 maintained or less likely to be used in the future (e.g., SOLiD and Roche 454),

79 the fact that large amounts of data have been produced under such

80 platforms means that NGS platform artifacts will need to be accounted for

81 within analyses that incorporate historical data. The rapid incorporation of

82 NGS data within many disciplines has produced many bioinformatic tools, all

83 of which may produce different results when trying to accomplish the same

84 task. For example, comparative studies have shown that not all de novo

85 assemblers are equal (Bradnam et al. 2013; Magoc et al. 2013; Salzberg et

86 al. 2012; Zhang et al. 2011) nor are mapping algorithms (Hatem et al. 2013;

87 Ruffalo et al. 2011) or variant detection algorithms (Cheng et al. 2014). As for

88 phylogenetic inference, performance comparisons of recently developed

89 maximum-likelihood methods to handle the large matrices often associated

90 with WGS data show that those methods do not always produce the same

91 topology (Liu et al. 2011).

92 In this study, our objective was to advance the use of WGS data within the

93 public health realm, and in general, by investigating the robustness of the

94 inferred phylogenetic relationships to several key factors that can vary

95 among analyses. Here, we define robustness as the sensitivity of results to

96 differences in the acquisition and analysis of data. In our case, data

97 acquisition and analysis represent the combination of different sequencing 
98 platforms, SNP detection methods, and phylogenetic inference algorithms;

99 the results are the inferred topology. We first present the reference-based

100 approach we have developed to identify SNPs among the WGS data we are

101 routinely producing. We then quantified the congruence among our method

102 and three other SNP detection methods (one reference-based and two

103 reference-free) by comparing the topologies produced with the same

104 phylogenetic inference package. We evaluated the influence of sequencing

105 platform by determining the degree to which replicate runs of the same strain

106 clustered together and the number of differences among replicate runs of the

107 same strain on the same platform. We also assessed the influence of three

108 different phylogenetic inference methods by comparing topologies created

109 using the same data matrix. These analyses were performed on a set of

110 Salmonella enterica subsp. enterica Serovar Montevideo isolates sequenced

111 on five different NGS platforms. Many of the isolates were implicated in an

112 outbreak of salmonellosis and the evolutionary relationships of a subset of

113 isolates were investigated within two previous studies (Allard et al. 2012; den

114 Bakker et al. 2011).

115 Materials and Methods

\section{Sequence Data}

117 All 194 samples included in this study were downloaded from the SRA archive

118 (Table S1) from which fastqs or sff files were extracted using the SRA Toolkit

119 v2.1.6. SOLiD SRA files were converted to colorspace fastq (csfastq). The 194

120 samples represent 107 unique strains, 3 of which are Salmonella enterica

121 ssp. enterica serovar Pomona that served as an outgroup and the others are

122 Montevideo (Table 1). Samples were from one of five platforms: Illumina

123 MiSeq and HiSeq, Life Technologies IonTorrent, Roche 454 FLX Titanium, and

124 Applied Biosystems SOLiD. Thirty-two strains were sequenced on more than

125 one sequencing technology (Tables $1 \&$ S1). All but the 454 runs for strain

126 IA_2009159199 are replicates from independent passages (overnight

127 cultures) and, thus, from different library preparations for sequencing.

128 In addition to the full dataset of 194 samples, we constructed a dataset

129 containing only those samples present in Allard et al. (2012). With the Allard

130 dataset (116 runs, 47 strains) our primary focus was on how the conclusions

131 within a traceback investigation (i.e., linking clinical isolates that sickened

132 people to environmental sources) would differ under the three factors being

133 considered (i.e., NGS platform, SNP detection method, and phylogenetic

134 inference method). The conclusions based on NGS data within that study are

135 also supported by epidemiological data. 
136 Given that we analyzed empirical data, we do not know the true evolutionary

137 relationships among the individuals. However, we do have replication of a

138 number of isolates and, therefore, have expectations for how those isolates

139 should cluster (i.e., be monophyletic) and the number of SNP differences that

140 should exist among them (i.e., zero). For analyses comparing topologies (see

141 below), we were not able to determine the accuracy of the methods but

142 rather focused on the variance among methods where we were working

143 under the assumption that methods that produced more similar trees are

144 superior to methods that result in different topologies.

\section{Variant Detection Methods}

146 We investigated the performance of four different SNP detection methods,

147 which can be broadly separated into reference-based and reference-free. The

148 primary difference between the two types is that reference-based methods

149 use a genome, either a closed or draft assembly, to which reads are mapped

150 and variant positions are called. In contrast, the reference-free approach

151 determines variant sites in the absence of a reference genome by comparing

152 portions (e.g., k-mers) among all samples.

153 Reference-based methods

154 For the reference-based methods we used the closed genome of strain

155 507440-20 that was sequenced on the PacBioRS II (Pacific Biosciences; Menlo

156 Park, CA, USA) and assembled using SMRT Analysis v2.0.1 (Genbank ID

157 CP007530.1). We chose this as the reference genome as it represented the

158 closest Montevideo environmental strain to the outbreak (Allard et al. 2012).

159 Raw reads from each sample were mapped to the reference genome using

160 default settings within Bowtie2 v2.1.0 (Fig. 1) (Langmead \& Salzberg 2012).

161 SOLiD data was mapped using Bowtie v1.0.0 (Langmead et al. 2009), which

162 has the ability to handle colorspace data. There are many other mappers that

163 could be used but we chose not to evaluate mappers as their performance

164 has been evaluated elsewhere (Hatem et al. 2013; Ruffalo et al. 2011). We

165 then used samtools v0.1.18 (Li et al. 2009) to sort the BAM file from Bowtie

166 and produce a pileup file for each sample.

167 Under our method, the resulting pileup files from samtools are processed

168 using VarScan2 v2.3 (Koboldt et al. 2012) to identify high quality variant sites

169 using the mpileup2snp option, which are stored in individual .vcf files. We

170 used the default values for variant detection except the minimum variant

171 allele frequency was set to 0.90 . As a result, we refer to this method as

172 VarScan. We used a custom python script to parse the .vcf files and construct

173 a SNP matrix, which included two steps. First, a 'snplist' file is generated by

174 identifying all variant sites across the individual .vcf files, which represents all

175 the positions that will make up the SNP matrix. Second, for each sample an 
176 additional pileup file is generated that contains only those positions found in

177 the 'snplist' file. It is from these second pileup files that we determine the

178 nucleotide state for each position in SNP matrix. Determining the state of

179 each position in the snplist file is based on the following rules: a) if different

180 nucleotides were called at the position, the one with frequency larger than

$18150 \%$ was the consensus call for that position but this threshold can be altered

182 to match a different user defined value; and b) if different nucleotides were

183 called at a position but none had a frequency larger than $50 \%$, that position

184 for that individual was coded as missing data. Positions identified as indels in

185 the .vcf file were ignored in the construction of the SNP matrix. Additional

186 information (e.g., our code and instructions) is at https://github.com/CFSAN-

187 Biostatistics/snp-pipeline.

188 To evaluate the sensitivity of our reference-based method, we also

189 constructed SNP matrices using the variant detection algorithm implemented

190 in SolSNP v1.11 (Fig. 1) (Robbins et al. 2011). Like our own method, reads

191 were mapped to a closed reference using Bowtiel or Bowtie2. However, in

192 contrast to our method that uses VarScan, the resulting bam files were then

193 processed with SolSNP, which uses a Kolmogorov-Smirnov statistic as a

194 distance measure to call the most likely nucleotide state with respect to the

195 reference. The resulting .vcf files were parsed using our python script to

196 construct the SNP matrices for downstream phylogenetic analyses. All SolSNP

197 analyses were conducted using the default parameters.

198 For the reference-based methods, in addition to the all matrix that consists of

199 all SNPs discovered in the analysis that had $0.7 \%$ missing data (Table 2), we

200 constructed a core matrix that consisted of only those SNP positions for

201 which every sample had a nucleotide called (Fig. 1).

202 Reference-free methods

203 We used the program KSNP v2.0 (Gardner \& Hall 2013) to produce SNP

204 matrices without using a reference sequence. KSNP uses a k-mer approach to

205 identify homologous single nucleotide polymorphisms among a group of

206 individuals. Briefly, the program uses jellyfish (Marcais \& Kingsford 2011) to

207 index all draft genomes into k-mers and SNPs are identified using MUMmer

208 (Kurtz et al. 2004). Although we are aware of at least one other k-mer based

209 approach, it requires paired-end data (Schwartz et al. 2013).

210 We used KSNP on two different treatments of the sequence data. Under the

211 first we used filtered fastq files; reads were filtered using seqtk

212 (https://github.com/lh3/seqtk), which masked bases with Q-scores $<20$.

213 Under the second treatment, we performed de novo assemblies that were

214 then used as the input for KSNP. We chose an assembler that was well suited 
215 for the sequencing platform from which the reads came, which was CLC

216 Genomics Workbench 6.0.5 (CLC Bio, Cambridge, MA, USA) for the Illumina, 217 SOLiD, and IonTorrent data and Newbler v2.6 for the 454 data. As was the 218 case with the mapping software under the reference-based approach, our

219 objective here was not to evaluate multiple de novo assemblers as that has 220 been done elsewhere (Magoc et al. 2013; Salzberg et al. 2012; Zhang et al. 221 2011). In both kSNP analyses, the k-mer size was set to 25.

222 For both the de novo and raw read approaches, three SNP matrices were 223 produced, an "all" matrix, a "majority" matrix, and a "core" matrix. The all 224 and core matrices are as defined for the reference-based methods. The 225 majority matrix contained only those positions in the all matrix for which at

226 least $50 \%$ of samples had a nucleotide base called. With the de novo

227 approach, the core matrix had 0 nucleotides present (likely due to missing

228 data associated with the poorer assemblies; Fig. 2) so we only analyzed the 229 all and majority matrix under that approach. The all matrix constructed with

230 the raw reads had $96 \%$ missing data and contained 2,990,475 positions

231 (Table 2), which does not seem biologically reasonable as it suggests that

232 that nearly $66 \%$ of the genome contains variable positions. As a result, we do

233 not perform any of the subsequent analyses on this matrix.

\section{Analyses of Replicate Runs}

235 Within each matrix, we calculated the proportional pairwise number of SNP

236 differences among replicates of a strain run on the same NGS platform.

237 Analyses were conducted using the dist.dna function within the ape package

238 (Paradis et al. 2004) in R (R Development Core Team 2011)

239 We used the genealogical sorting index (gsi) (Cummings et al. 2008) to 240 quantify the degree to which replicate runs of the same strain clustered 241 together, which provides insight into the robustness of the phylogenetic 242 relationships to differences in sequencing platform and bioinformatic 243 approaches. The gsi varies from 0 (a completely random assortment of 244 individuals from the same group (e.g., strain) on the tree) to 1 (replicates are 245 reciprocally monophyletic). Generally speaking, the gsi is based on the 246 number of nodes uniting all individuals from the same group divide by the 247 observed number of nodes uniting those members. We calculated the gsi 248 statistic for two different cases: 1) all replicates of a given strain comprise a 249 single group and 2) replicates run on the same platform comprise a group. 250 We chose these two groupings because they allowed us to characterize, as 251 best we could given the dataset, the influence of within and between 252 platform variation. To account for phylogenetic uncertainty, we estimated the 253 weighted gsi value under which 100 bootstrap replicates for each matrix 
254 (e.g., VarScan, KSNP de novo) by phylogenetic inference comparison were 255 analyzed.

\section{Phylogenetic Analysis}

257 Topologies based on each of the matrices created under the different SNP

258 detection methods were created using three phylogenetic inference methods

259 (FastTreeMP (Price et al. 2010), GARLI (Zwickl 2006), and RaxML (Stamatakis

260 2006)). Each of the three methods uses a different approach to increase

261 computational feasibility when analyzing large matrices. When evaluating the

262 robustness of the results to different SNP detection methods or NGS

263 platforms, we only compared topologies produced using the same

264 phylogenetic inference method. To assess the robustness of results to

265 differences in phylogenetic inference method, we only compared topologies

266 constructed with the same SNP matrix.

267 For the FastTreeMP v2.1.7 analyses, we used the - gtr - nt - cat 4 flags;

268 analyses were run on a single desktop machine with dual $2.93 \mathrm{GHz} 6$-Core

269 Intel Xeon processors and 48 GB of shared RAM. We used phylip's seqboot

270 (Felsenstein 1989) option to produce 100 bootstrap replicates of the dataset,

271 each of which were then analyzed with FastTreeMP as was done with the non-

272 bootstrapped dataset. Given that the other two methods were carried out

273 conducting traditional non-parametric bootstrapping we thought it

274 appropriate to do the same with FastTreeMP rather than rely on the SH-like

275 local support values that program can also produce. We used the default

276 parameter setting for the GARLI V2.0 analyses including the GTR $+I+\Gamma$. We

277 ran 100 replicate GARLI analyses for each of the observed matrices, and

278 present the topology with the best likelihood score to which support values

279 based on 1000 bootstrap replicates were added. GARLI analyses were

280 performed on a high performance computer within the FDA's Scientific

281 Computing Lab; batches of up to 20 runs were performed simultaneously on

282 this resource where compute nodes contain 8 Intel Xeon $2.67 \mathrm{GHz}$ processors

283 and 24 GB of shared RAM. For RAXML V7.9.1, we used raxmIHPC-PTHREADS-

284 AVX, the - $m$ GTRCAT flag, and - b flag to perform 1000 non-parametric

285 standard bootstrap replicates; analyses were run on a Linux machine with 32

286 Intel Xeon $2.00 \mathrm{GHz}$ processors and $64 \mathrm{~GB}$ of shared RAM.

287 Topological Congruence

288 We quantified the congruence among topologies using the symmetric 289 difference statistic (Steel \& Penny 1993) (i.e, the Robinson-Foulds distance 290 (Robinson \& Foulds 1981)) implemented in the phangorn package (Schliep 291 2011) in R. The symmetric difference statistic is twice the number of internal 292 nodes that differ in their branching between two trees. Our interpretations of

293 the symmetric difference statistic are based on the assumption that methods 
294 or matrix types that result in similar topologies are superior to those that do

295 not. As was the case with the gsi analyses, to account for phylogenetic

296 uncertainty we calculated the symmetric difference between all pairwise

297 comparisons of 100 bootstrap replicates (e.g., pairwise comparisons between

298100 GARLI bootstrap replicates of the VarScan and KSNP de novo matrices).

\section{Results}

\section{Mapping and de novo assembly summary statistics}

301 Although the number and lengths of reads varied greatly among the 302 platforms, at least $50 \%$ of the reads could be mapped for all but a few 303 samples (Fig. 2). The number of contigs and N50 values varied among 304

305 platforms but for the majority of the samples, except perhaps the SOLiD runs, the length of the assemblies were close to the expected 4.5 Mbp genome size of a $S$. Montevideo (Fig. 2). These mapping and assembly summary statistics are promising in that they suggest that similar amounts of data can be

\section{Platform effects}

310 To characterize platform variability, we examined replicate runs of the same 311 sample sequenced on the same platform (Fig. 3). Ideally these replicates 312 would have no proportional pairwise differences and be identical. In reality 313 we found that the 454 platform had the greatest number of differences, 314 followed by Ion Torrent, and then by MiSeq. This pattern was generally 315 consistent across the different variant detection methods except for the KSNP 316 de novo datasets where lonTorrent replicates were more different from one 317 another than on the 454 platform. (The SOLiD platform had no replicate runs 318 and was not assessed). We note that many of the replicate samples are not 319 pure platform replicates, but were from independent cultures often performed 320 at different labs, which means our observed differences are not just due to 321 platform variability.

322 To examine the effect of platform on clustering, we computed the weigthed 323 gsi with classes assigned by strain and with classes assigned by the 324 combination of strain and platform. Regardless of matrix type and SNP 325 detection method, the strain replicates did not cluster together and, thus, gsi 326 values for many strain classes were $<1$ (Fig. 4). When classes were defined 327 by the combination of strain and platform, there was a much higher average 328 gsi score. This implies that replicates do cluster together more reliably when 329 run on the same platform. However, despite the differences in reproducibility 330 and gsi values between the classes, we did not find evidence to suggest that 331 there is a strong platform effect when clustering (e.g., clades were not found 
332 consisting of only samples run on a single platform; Supplemental Data Fig.

$333 \mathrm{~S} 1)$.

\section{Matrix size effects}

335 Our results indicate that a matrix that accepts some level of missing data, 336 while not including all sites for which only a few samples have nucleotides

337 states is optimal for phylogenetic inference. For example, topologies created

338 using core matrices within which there is no missing data had a greater 339 degree of topological incongruence (Fig. 5), greater number of identical

340 sequences (Table 2), and poorer phylogenetic resolution (Fig. 6) when

341 compared to topologies inferred with non-core matrices. However, the all

342 SNP matrix created using the reference-free method with de novo assemblies

343 had approximately 20,000 more SNPs compared to the majority matrices

344 under the reference-free approaches and the all matrices under the

345 reference-based methods, which is an increase in matrix size of

346 approximately $30 \%$. Although some of these additional SNPs may be due to

347 mobile elements/gene presence-absence, it is also likely that many of them

348 are erroneous, which may explain the decrease in bootstrap support despite

349 the larger matrix when compared to the de novo majority matrix (Fig. 6). An

350 additional explanation for the decreased support is that the larger matrices

351 also have more missing data that may increase phylogenetic uncertainty

352 (Roure et al. 2013). The higher proportional differences among replicates

353 observed in this matrix also suggests that the additional SNPs may be

354 erroneous and, thus, such matrices should be avoided (Fig. 3). In conclusion,

355 within a reference-based approach, it is probably optimal to use all SNP sites

356 detected rather than cull positions with missing data; for a reference-free

357 approach the threshold of missing data that results in optimal information

358 content is more complicated and depends, in part, on the evolutionary

359 breadth of the samples being investigated.

360 Differences due to SNP detection method

361 The proportion of SNP differences among replicate samples run on the same

362 platform varied, although not substantially, across the matrices created

363 under either the reference-free or reference-based approach (Fig. 3). Given

364 that all methods are 1 ) working with the same raw reads and 2) either the

365 same assemblies or bam files for kSNP de novo and reference based

366 approaches, respectively, these differences are predominantly due to the

367 differences in algorithms used to identify variant sites.

368 The method used to construct the SNP matrix had a significant effect on the

369 degree of congruence among trees (Figs. $5 \& 7$ ). For example, trees

370 constructed using a reference-based method were more similar to one

371 another (i.e., had a lower symmetric difference score) than those produced 
372 under a reference-free approach or when comparing trees created under the 373 different methods.

\section{Phylogenetic inference method}

375 For both the reference-free and reference-based approaches there was 376 greater topological congruence when comparing a tree produced under 377 RAxML and GARLI than when either of the trees produced under those 378 methods were compared to one inferred with FastTreeMP. For example, more 379 topological rearrangements are required to reconcile FastTreeMP trees with 380 either GARLI or RAXML (Fig. 7). As we do not know the true topology, we note 381 that when comparing the different inference methods we are working under 382 the assumption that methods that result in similar topologies are optimal. 383 This also means that we cannot assess the accuracy of the methods but, 384 rather, we are estimating the variance among the topological differences 385 they produce.

\section{Allard et al. (Allard et al. 2012) samples}

To explore the consequences of differences observed among topologies as a result of a combination of NGS platform and SNP detection method within an outbreak scenario, we pared down the dataset to include the 116 runs that represented only the 47 strains present in Allard et al. (Allard et al. 2012). Here, we refer to outbreak isolates as those whose phylogenetic placement clusters them with the clinical samples from infected individuals and nonoutbreak samples are those whose topological placement is outside of the cluster containing the focal clinical samples. We focused on whether outbreak runs clustered together; if they did, that would provide support that significant patterns related to outbreak investigations are robust to combining runs across different sequencing platforms and using different SNP detection and/or phylogenetic inference packages.

When using RAXML or GARLI to infer a topology, the VarScan, SoISNP, KSNP de novo and KSNP raw matrices constructed with the Allard et al. (Allard et al. 2012) dataset resulted in strong support (100 to 81 BP (bootstrap probability); Table 3 ) differentiating non-outbreak from outbreak isolates that were consistent with the results of that study (Fig. 8). The de novo referencefree approach had the lowest level of bootstrap support among both the GARLI $(B P=81)$ and RAxML $(B P=91)$ trees. The results using FastTreeMP were only congruent with the previous results when analyzing the KSNP de novo, KSNP all and VarScan core matrices, the latter two had low support ( $\leq$ 65 BP) (Table 3; Supplemental Data Fig. S2). Interestingly, we found that both reference-based all matrices had six fixed SNP differences that differentiated 411 the outbreak and non-outbreak clades; there was only a single fixed SNP difference within the reference-free majority matrices. The difference 
412 between the two methods is due to the exclusion under the reference-free 413 approach of SNPs that are within the k-mer distance of one another (i.e., 25 414 bp).

\section{Discussion}

416 Although the performance of NGS platforms and the requisite analysis

417 software has been evaluated, those evaluations were often done focusing on

418 a single step in the process (e.g., mapping, de novo assembly, or

419 phylogenetic inference). Here, we have considered the entire process from

420 the sequence platform to phylogenetic clustering. Examination of the full

421 process is important in elucidating best practices to increase the consistency

422 and accuracy of such analyses. We found that within each of the three factors

423 we evaluated there were differences in the results produced and, thus, were

424 able to identify some guidelines for how NGS data should be analyzed for the

425 purpose of clustering.

\section{Variability among NGS platforms}

427 Ideally, different next-generation sequencers would produce identical

428 nucleotide sequences when provided with the same sample. Unsurprisingly

429 however, differences in the chemistry and engineering of different platforms

430 mean they do not agree exactly, but have different error rates and error

431 structure. For example, studies of "benchtop" NGS platforms have repeatedly

432 found that the MiSeq has the lowest single-base error rate followed by

433 Roche's 454 GS Junior followed by the Ion Torrent PGM (Junemann et al. 2013;

434 Loman et al. 2012; Quail et al. 2012); unlike the MiSeq, homopolymer-

435 associated indel errors were an artifact of the latter two platforms (Loman et

436 al. 2012). SOLiD, with much smaller read lengths than the other platforms

437 investigated, had the lowest sequencing accuracy, lowest coverage rate, and

438 highest false-positive rate when compared to Roche 454 and Illumina GA data

439 (Harismendy et al. 2009).

440 Given the differences between NGS platforms, we must ask if the differences

441 are significant enough to obscure the relationships among individuals. From

442 our analysis of sequencing runs of the same strain carried out on different

443 platforms and labs, it is clear that replicate runs were not identical (Fig. 3)

444 and, consequently, replicates did not always result in a monophyletic clade

445 (Fig. 4). However, replicates of a strain sequenced on the same platform were 446 more likely to represent such a clade (Fig. 4). And although replicates did not 447 always cluster together they were not likely to be found in different strongly

448 supported clades. These results suggest that it would be ideal to analyze 
449 samples sequenced on a single NGS platform but that platform variation is unlikely to result in strongly supported erroneous relationships.

\section{Reference-free vs. reference-based approaches}

452 The analysis of WGS is non-trivial, and there are a number of possible 453 approaches that exist. Here we focused on one possible way that such data 454 could be used: to identify variant sites to construct a SNP matrix from which a 455 phylogeny could be inferred. Some of the approaches (e.g., Mauve; (Darling 456 et al. 2010)) that can accomplish this are applicable for smaller data sets as 457 they are based on whole genome alignments and were not considered here 458 as we wish to construct a SNP matrix and a resulting phylogeny for 100s 4591,000 s of runs. We considered a number of methods that are suitable for 460 large datasets (i.e., reference-free and reference-based) and, therefore, likely 461 represent those most likely to be employed in future studies.

462 We use a reference-based method in our outbreak investigations because it 463 was assumed to have lower false-discovery rate and naturally excludes 464 variants within the mobilome (e.g., prophages and plasmids), which the 465 results presented here support. However, the primary drawback of a 466 reference-based method is that it will fail to detect SNPs within a region 467 shared among non-reference samples but that is absent in the reference 468 genome either due to using a reference whose genome has not been closed 469 (e.g., an incomplete draft assembly) or the genomic region actually being 470 absent in the reference. In contrast, the reference-free approach will detect 471 SNPs within regions missing from a subset of the runs (depending on the 472 percent presence threshold). This additional detection ability comes at the 473 cost of having to decide what threshold one should use; we used the $50 \%$ 474 majority but other values could include the percent of the dataset that 475 represents the ingroup samples. Without a threshold on the percent of 476 missing data, the reference-free approach may have a much higher false477 discovery rate most likely due to sequencing error (e.g., jackpot mutations;

478 (Kraytsberg \& Khrapko 2005)), which is well illustrated by the all matrix using 479 raw reads having approximately three million basepairs. However, even when 480 minimizing the number of false positive SNPs in the matrix through a 481 threshold, the reference-free method still produced more incongruent trees 482 than the matrices constructed with the reference-based methods (Fig. 5).

\section{Phylogenetic inference}

484 The three phylogenetic inference methods we evaluated did not produce the 485 same topology when analyzing the same SNP matrix (Fig. 7). However, many 486 of the topological differences were likely among poorly supported clades (Fig. 487 5). The topologies produced with RAXML and GARLI did recover the general 488 clustering pattern that differentiates outbreak from non-outbreak isolates 
489 more frequently than FastTreeMP. This result is consistent with previous work 490 (Liu et al. 2011) indicating that RAxML has higher accuracy than FastTreeMP. 491 (This does come with a speed trade-off. For the 194 sample dataset with 492 approximately 50000 bp per sample analyzed here, a phylogeny could be 493 inferred with FastTreeMP in a matter of minutes and for RAXML and GARLI it 494 was on the order of hours and days, respectively, to obtain a topology and 495 bootstrap replicates.) RAXML and GARLI topologies were fairly congruent with 496 one another for strongly supported branch-points. As a result, for closely 497 related organisms like those studied here there may be a real cost in using 498 faster but less thorough search algorithms.

499 An important aspect of our phylogenetic analyses is that the substitution 500 models we employed may not be optimal for the types of matrices we were 501 analyzing within which every site is variable. To our knowledge there have 502 been few studies investigating the effects of applying traditional nucleotide 503 substitution models to such matrices. One such study found that under the 504 GTR + $\Gamma$ model, phylogenetic accuracy decreased when invariant sites were 505 excluded (Bertels et al. 2014). Although these SNP matrices may be better 506 modeled as binary data to which the Mk or Mkv models (Lewis 2001) could be 507 applied. However, that model is computationally demanding and may not be 508 well suited for the large matrices associated with WGS data; it also has a 509 number of assumptions that SNP matrices likely violate (e.g., an infinite sites 510 model and equal substitution rates between states). Within the RAxML

511 package, the ASC_GTRGAMMA option which models no invariant sites and, 512 thus, may be appropriate but that model also corrects for ascertainment bias, 513 which is not appropriate for the matrices we constructed. Given this potential 514 for model misspecification, an avenue of future research that would 515 strengthen the robustness of inferring phylogenies from SNP matrices would 516 be to determine the consequences of model violations and the development 517 of more appropriate nucleotide substitution models for such matrices

\section{Signal vs. noise}

519 We have evaluated the robustness of topologies to a number of factors that 520 may differ among analyses on a particularly vexing group of samples that are 521 highly clonal and very closely related. Within the original analyses describing 522 the relationships among 47 of the strains, there were only five unique SNP 523 differences between outbreak and non-outbreak isolates (Allard et al. 2012). 524 Within this study the reference-based non-core matrices had six fixed SNP 525 differences while the reference-free approaches had only one. This raises the 526 question of whether there is the possibility that within the current study 527 noise, either due to sequencing errors, variation in SNP detection method, 528 and/or phylogenetic inference, would render impossible our ability to recover 529 the outbreak/non-outbreak structure. Such issues are less likely to arise when 
530 investigating a greater degree of evolutionary divergence assuming that a

531 sufficient number of homologous sites can be identified since there will be

532 many more real SNP differences and, thus, a single topology is likely to

533 emerge from different methods. However, due to a number of issues (e.g.,

534 lack of a suitable reference genome and difficulties detecting k-mers present

535 in all samples) there will also be an upper bound to the evolutionary breadth

536 of relationships that can be investigated with the methods we have

537 evaluated.

538 The results presented here indicate it is possible to recover the outbreak 539 structure in a data set of this type, but care should be taken when deciding

540 on the methods to employ. Methodological choices include: 1) deciding to

541 combine data from different NGS platforms, 2) choosing a method to use for

542 identifying variants, and 3) choosing what phylogenetic inference method

543 should be used. Our results suggest good options for each of these choices

544 and indicate that they allow detection of the biological outbreak signal even

545 among these closely related samples. For example, we found strong

546 bootstrap support for the differentiation of outbreak and non-outbreak

547 samples results when using the non-core matrices constructed using a

548 reference-based method and inferring a phylogeny with either RAxML or

549 GARLI (Table 3). Although we did not evaluate it here, we note that care

550 should also be taken regarding the choice of a reference, as there are a

551 number of studies that have found that topological accuracy and the number

552 of erroneous SNP detected depends on the reference chosen (Bertels et al.

553 2014; Pightling et al. 2014).

\section{Conclusions}

555 The robustness of phylogenetic inference to data acquisition and analysis

556 methodology is critically important in many studies (e.g., traceback

557 investigations, systematics, and phylogeography). Our results illustrate how

558 topologies may differ depending on methodological approaches employed - a

559 particularly important issue when investigating closely related samples. Our

560 results also show that good methods do exist that provide robust results

561 when attempting to differentiate between outbreak and non-outbreak

562 samples. For example, the best choice appears to be the use a reference-

563 based approach allowing for some missing data in the matrix and

564 constructing trees using a thorough inference program. We believe that these

565 general conclusions are likely to be observed across different datasets (e.g.,

566 within other taxonomic groups and across a range of genome complexity)

567 than the one investigated here and future studies will reveal whether that

568 assumption is correct. 
569 This work represents our ongoing efforts to build, characterize, and validate

570 our WGS methodology and pipeline for food-borne outbreak investigations.

571 The questions we address are applicable to many in the WGS analysis

572 community, and we hope that the results presented here will contribute to

573 the establishment, through additional investigations, of well-defined pipelines

574 for clustering based on WGS data.

\section{Acknowledgements}

576 We thank Cong $\mathrm{Li}$ and Charles Wang for helping to produce whole genome

577 sequence data. We would like to acknowledge Henk den Bakker and Martin

578 Weidman from Cornell University; and Furtado Manohar, Craig Cummings,

579 and Lavorka Degoticija from LifeTech for producing the SOLiD and some of

580 the lonTorrent data. We also acknowledge the staff at the FDA's Scientific

581 Computing Lab for assistance with running analyses on the HPC. We thank

582 Eric Brown, Peter Evans and Steve Musser for promoting this research. 
584

585

586

587

588

589

590

591

592

593

594

595

596

597

598

599

600

601

602

603

604

605

606

607

608

609

610

611

612

613

614

615

616

617

618

619

620

621 Cummings MP, Neel MC, and Shaw KL. 2008. A genealogical approach to

622

Allard MW, Luo Y, Strain E, Li C, Keys CE, Son I, Stones R, Musser SM, and Brown EW. 2012. High resolution clustering of Salmonella enterica serovar Montevideo strains using a next-generation sequencing approach. BMC Genomics 13:32.

Bertels F, Silander OK, Pachkov M, Rainey PB, and van Nimwegen E. 2014. Automated Reconstruction of Whole-Genome Phylogenies from Short-Sequence Reads. Molecular Biology and Evolution 31:10771088.

Bielaszewska M, Mellmann A, Zhang WL, Kock R, Fruth A, Bauwens A, Peters G, and Karch H. 2011. Characterisation of the Escherichia coli strain associated with an outbreak of haemolytic uraemic syndrome in Germany, 2011: a microbiological study. Lancet Infectious Diseases 11:671-676.

Bradnam KR, Fass JN, Alexandrov A, Baranay P, Bechner M, Birol I, Boisvert S, Chapman JA, Chapuis G, Chikhi R, Chitsaz H, Chou WC, Corbeil J, Del Fabbro C, Docking TR, Durbin R, Earl D, Emrich S, Fedotov $P$, Fonseca NA, Ganapathy G, Gibbs RA, Gnerre S, Godzaridis E, Goldstein S, Haimel M, Hall G, Haussler D, Hiatt JB, Ho IY, Howard J, Hunt M, Jackman SD, Jaffe DB, Jarvis ED, Jiang $H$, Kazakov S, Kersey PJ, Kitzman JO, Knight JR, Koren S, Lam TW, Lavenier D, Laviolette F, Li Y, Li Z, Liu B, Liu Y, Luo R, Maccallum I, Macmanes MD, Maillet N, Melnikov S, Naquin D, Ning Z, Otto TD, Paten B, Paulo OS, Phillippy AM, Pina-Martins F, Place M, Przybylski D, Qin X, Qu C, Ribeiro FJ, Richards S, Rokhsar DS, Ruby JG, Scalabrin S, Schatz MC, Schwartz DC, Sergushichev A, Sharpe T, Shaw TI, Shendure J, Shi Y, Simpson JT, Song H, Tsarev F, Vezzi F, Vicedomini R, Vieira BM, Wang J, Worley KC, Yin S, Yiu SM, Yuan J, Zhang G, Zhang H, Zhou S, and Korf IF. 2013. Assemblathon 2: evaluating de novo methods of genome assembly in three vertebrate species. Gigascience 2:10.

Cheng AY, Teo YY, and Ong RTH. 2014. Assessing single nucleotide variant detection and genotype calling on whole-genome sequenced individuals. Bioinformatics 30:1707-1713.

Chin CS, Sorenson J, Harris JB, Robins WP, Charles RC, Jean-Charles RR, Bullard J, Webster DR, Kasarskis A, Peluso P, Paxinos EE, Yamaichi Y, Calderwood SB, Mekalanos JJ, Schadt EE, and Waldor MK. 2011. The Origin of the Haitian Cholera Outbreak Strain. New England Journal of Medicine 364:33-42. quantifying lineage divergence. Evolution 62:2411-2422. 
623

624

625

626

627

628

629

630

631

632

633

634

635

636

637

638

639

640

641

642

643

644

645

646

647

648

649

650

651

652

653

654

655

656

657

658

659

660

661

662

den Bakker HC, Switt AIM, Cummings CA, Hoelzer K, Degoricija L, RodriguezRivera LD, Wright EM, Fang RX, Davis M, Root T, Schoonmaker-Bopp D, Musser KA, Villamil E, Waechter H, Kornstein L, Furtado MR, and Wiedmann M. 2011. A Whole-Genome Single Nucleotide Polymorphism-Based Approach To Trace and Identify Outbreaks Linked to a Common Salmonella enterica subsp enterica Serovar Montevideo Pulsed-Field Gel Electrophoresis Type. Applied and Environmental Microbiology 77:8648-8655.

Felsenstein J. 1989. PHYLIP - Phylogeny Inference Package (Version 3.2). Cladistics 5:164-166.

Gardner SN, and Hall BG. 2013. When Whole-Genome Alignments Just Won't Work: KSNP v2 Software for Alignment-Free SNP Discovery and Phylogenetics of Hundreds of Microbial Genomes. PLoS One 8:e81760.

Harismendy O, Ng PC, Strausberg RL, Wang XY, Stockwell TB, Beeson KY, Schork NJ, Murray SS, Topol EJ, Levy S, and Frazer KA. 2009. Evaluation of next generation sequencing platforms for population targeted sequencing studies. Genome Biol 10.

Hatem A, Bozdag D, Toland AE, and Catalyurek UV. 2013. Benchmarking short sequence mapping tools. BMC Bioinformatics 14.

Hendriksen RS, Price LB, Schupp JM, Gillece JD, Kaas RS, Engelthaler DM, Bortolaia V, Pearson T, Waters AE, Upadhyay BP, Shrestha SD, Adhikari S, Shakya G, Keim PS, and Aarestrup FM. 2011. Population genetics of Vibrio cholerae from Nepal in 2010: evidence on the origin of the Haitian outbreak. Mbio 2:e00157-00111.

Junemann S, Sedlazeck FJ, Prior K, Albersmeier A, John U, Kalinowski J, Mellmann A, Goesmann A, von Haeseler A, Stoye J, and Harmsen D. 2013. Updating benchtop sequencing performance comparison. Nature Biotechnology 31:294-296.

Keim PS, Aarestrup FM, Shakya G, Price LB, Hendriksen RS, Engelthaler DM, and Pearson T. 2011. Reply to "South Asia Instead of Nepal May Be the Origin of the Haitian Cholera Outbreak Strain". Mbio 2.

Koboldt DC, Zhang QY, Larson DE, Shen D, McLellan MD, Lin L, Miller CA, Mardis ER, Ding L, and Wilson RK. 2012. VarScan 2: Somatic mutation and copy number alteration discovery in cancer by exome sequencing. Genome Res 22:568-576.

Koser CU, Holden MT, Ellington MJ, Cartwright EJ, Brown NM, Ogilvy-Stuart AL, Hsu LY, Chewapreecha C, Croucher NJ, Harris SR, Sanders M, Enright MC, Dougan G, Bentley SD, Parkhill J, Fraser LJ, Betley JR, SchulzTrieglaff OB, Smith GP, and Peacock SJ. 2012. Rapid whole-genome 
663

664

665

666

667

668

669

670

671

672

673

674

675

676

677

678

679

680

681

682

683

684

685

686

687

688

689

690

691

692

693

694

695

696

697

698

699

sequencing for investigation of a neonatal MRSA outbreak. $N$ Engl J Med 366:2267-2275.

Kraytsberg Y, and Khrapko K. 2005. Single-molecule PCR: an artifact-free PCR approach for the analysis of somatic mutations. Expert Review of Molecular Diagnostics 5:809-815.

Kurtz S, Phillippy A, Delcher AL, Smoot M, Shumway M, Antonescu C, and Salzberg SL. 2004. Versatile and open software for comparing large genomes. Genome Biol 5:R12.

Langmead B, and Salzberg SL. 2012. Fast gapped-read alignment with Bowtie 2. Nature Methods 9:357-U354.

Langmead B, Trapnell C, Pop M, and Salzberg SL. 2009. Ultrafast and memory-efficient alignment of short DNA sequences to the human genome. Genome Biol 10.

Lewis PO. 2001. A likelihood approach to estimating phylogeny from discrete morphological character data. Systematic Biology 50:913-925.

Li H, Handsaker B, Wysoker A, Fennell T, Ruan J, Homer N, Marth G, Abecasis G, Durbin R, and Proc GPD. 2009. The Sequence Alignment/Map format and SAMtools. Bioinformatics 25:2078-2079.

Lienau EK, Strain E, Wang C, Zheng J, Ottesen AR, Keys CE, Hammack TS, Musser SM, Brown EW, Allard MW, Cao G, Meng J, and Stones R. 2011. Identification of a salmonellosis outbreak by means of molecular sequencing. $N$ Engl J Med 364:981-982.

Liu K, Linder CR, and Warnow T. 2011. RAxML and FastTree: Comparing Two Methods for Large-Scale Maximum Likelihood Phylogeny Estimation. PLoS One 6.

Loman NJ, Misra RV, Dallman TJ, Constantinidou C, Gharbia SE, Wain J, and Pallen MJ. 2012. Performance comparison of benchtop highthroughput sequencing platforms (vol 30, pg 434, 2012). Nature Biotechnology 30:562-562.

Magoc T, Pabinger S, Canzar S, Liu XY, Su Q, Puiu D, Tallon LJ, and Salzberg SL. 2013. GAGE-B: an evaluation of genome assemblers for bacterial organisms. Bioinformatics 29:1718-1725.

Marcais G, and Kingsford C. 2011. A fast, lock-free approach for efficient parallel counting of occurrences of k-mers. Bioinformatics 27:764770.

Mardis ER. 2013. Next-generation sequencing platforms. Annu Rev Anal Chem (Palo Alto Calif) 6:287-303. 
700

701

702

703

704

705

706

707

708

709

710

711

712

713

714

715

716

717

718

719

720

721

722

723

724

725

726

727

728

729

730

731

732

733

734

735

736
Mellmann A, Harmsen D, Cummings CA, Zentz EB, Leopold SR, Rico A, Prior K, Szczepanowski R, Ji YM, Zhang WL, McLaughlin SF, Henkhaus JK, Leopold B, Bielaszewska M, Prager R, Brzoska PM, Moore RL, Guenther S, Rothberg JM, and Karch H. 2011. Prospective Genomic Characterization of the German Enterohemorrhagic Escherichia coli O104:H4 Outbreak by Rapid Next Generation Sequencing Technology. PLoS One 6.

Paradis E, Claude J, and Strimmer K. 2004. APE: Analyses of Phylogenetics and Evolution in R language. Bioinformatics 20:289-290.

Parkhill J, and Wren BW. 2011. Bacterial epidemiology and biology - lessons from genome sequencing. Genome Biol 12.

Pightling AW, Petronella N, and Pagotto F. 2014. Choice of Reference Sequence and Assembler for Alignment of Listeria monocytogenes Short-Read Sequence Data Greatly Influences Rates of Error in SNP Analyses. PLoS One 9:e104579.

Price MN, Dehal PS, and Arkin AP. 2010. FastTree 2 -- Approximately maximum-likelihood trees for large alignments. PLoS One 5:e9490.

Quail MA, Smith M, Coupland P, Otto TD, Harris SR, Connor TR, Bertoni A, Swerdlow HP, and Gu Y. 2012. A tale of three next generation sequencing platforms: comparison of Ion Torrent, Pacific Biosciences and Illumina MiSeq sequencers. BMC Genomics 13.

R Development Core Team. 2011. R: A language and environment for statistical computing. Vienna, Austria: R Foundation for Statistical Computing.

Robbins CM, Tembe WA, Baker A, Sinari S, Moses TY, Beckstrom-Sternberg S, Beckstrom-Sternberg J, Barrett M, Long J, Chinnaiyan A, Lowey J, Suh E, Pearson JV, Craig DW, Agus DB, Pienta KJ, and Carpten JD. 2011. Copy number and targeted mutational analysis reveals novel somatic events in metastatic prostate tumors. Genome Res 21:4755 .

Robinson DF, and Foulds LR. 1981. Comparison of Phylogenetic Trees. Mathematical Biosciences 53:131-147.

Roetzer A, Diel R, Kohl TA, Ruckert C, Nubel U, Blom J, Wirth T, Jaenicke S, Schuback S, Rusch-Gerdes S, Supply P, Kalinowski J, and Niemann S. 2013. Whole Genome Sequencing versus Traditional Genotyping for Investigation of a Mycobacterium tuberculosis Outbreak: A Longitudinal Molecular Epidemiological Study. Plos Medicine 10. 
737

738

739

740

741

742

743

744

745

746

747

748

749

750

751

752

753

754

755

756

757

758

759

760

761

762

763

764

765

766

767

768

769

770

771

772
Roure B, Baurain D, and Philippe H. 2013. Impact of Missing Data on Phylogenies Inferred from Empirical Phylogenomic Data Sets. Molecular Biology and Evolution 30:197-214.

Ruffalo M, LaFramboise T, and Koyuturk M. 2011. Comparative analysis of algorithms for next-generation sequencing read alignment. Bioinformatics 27:2790-2796.

Salzberg SL, Phillippy AM, Zimin A, Puiu D, Magoc T, Koren S, Treangen TJ, Schatz MC, Delcher AL, Roberts M, Marcais G, Pop M, and Yorke JA. 2012. GAGE: A critical evaluation of genome assemblies and assembly algorithms. Genome Res 22:557-567.

Schliep KP. 2011. phangorn: phylogenetic analysis in R. Bioinformatics 27:592-593.

Schwartz RS, Harkins K, Stone AC, and Cartwright RA. 2013. SISRS: SNP Identification from Short Read Sequences. arXiv 1305.3665.

Shendure J, and Ji HL. 2008. Next-generation DNA sequencing. Nature Biotechnology 26:1135-1145.

Snitkin ES, Zelazny AM, Thomas PJ, Stock F, Group NCSP, Henderson DK, Palmore TN, and Segre JA. 2012. Tracking a hospital outbreak of carbapenem-resistant Klebsiella pneumoniae with whole-genome sequencing. Sci Transl Med 4:148ra116.

Stamatakis A. 2006. RAxML-VI-HPC: Maximum likelihood-based phylogenetic analyses with thousands of taxa and mixed models. Bioinformatics 22:2688-2690.

Steel MA, and Penny D. 1993. Distributions of Tree Comparison Metrics Some New Results. Systematic Biology 42:126-141.

Underwood AP, Dallman T, Thomson NR, Williams M, Harker K, Perry N, Adak B, Willshaw G, Cheasty T, Green J, Dougan G, Parkhill J, and Wain J. 2013. Public Health Value of Next-Generation DNA Sequencing of Enterohemorrhagic Escherichia coli Isolates from an Outbreak. Journal of Clinical Microbiology 51:232-237.

Zhang WY, Chen J, Yang Y, Tang YF, Shang J, and Shen BR. 2011. A Practical Comparison of De Novo Genome Assembly Software Tools for NextGeneration Sequencing Technologies. PLoS One 6.

Zwickl DJ. 2006. Genetic algorithm approaches for the phylogenetic analysis of large biological sequence ddatasets under the maximumlikelihood criterion Ph. D. The University of Texas at Austin. 
PeerJ reviewing PDF | (v2014:04:1996:2:0:NEW 19 Sep 2014) 


\section{Figure 1}

Figure 1. Workflow describing the analyses conducted in this study.

Dotted boxes highlight the four effects (sequencing platform, SNP detection method, and phylogenetic inference) that are accounted for at that step in the process. The platforms and methods we currently use in our investigations are in bold italic font. 
Platform effects

SRA Data

(454, HiSeq, IonTorrent, MiSeq, SOLiD)

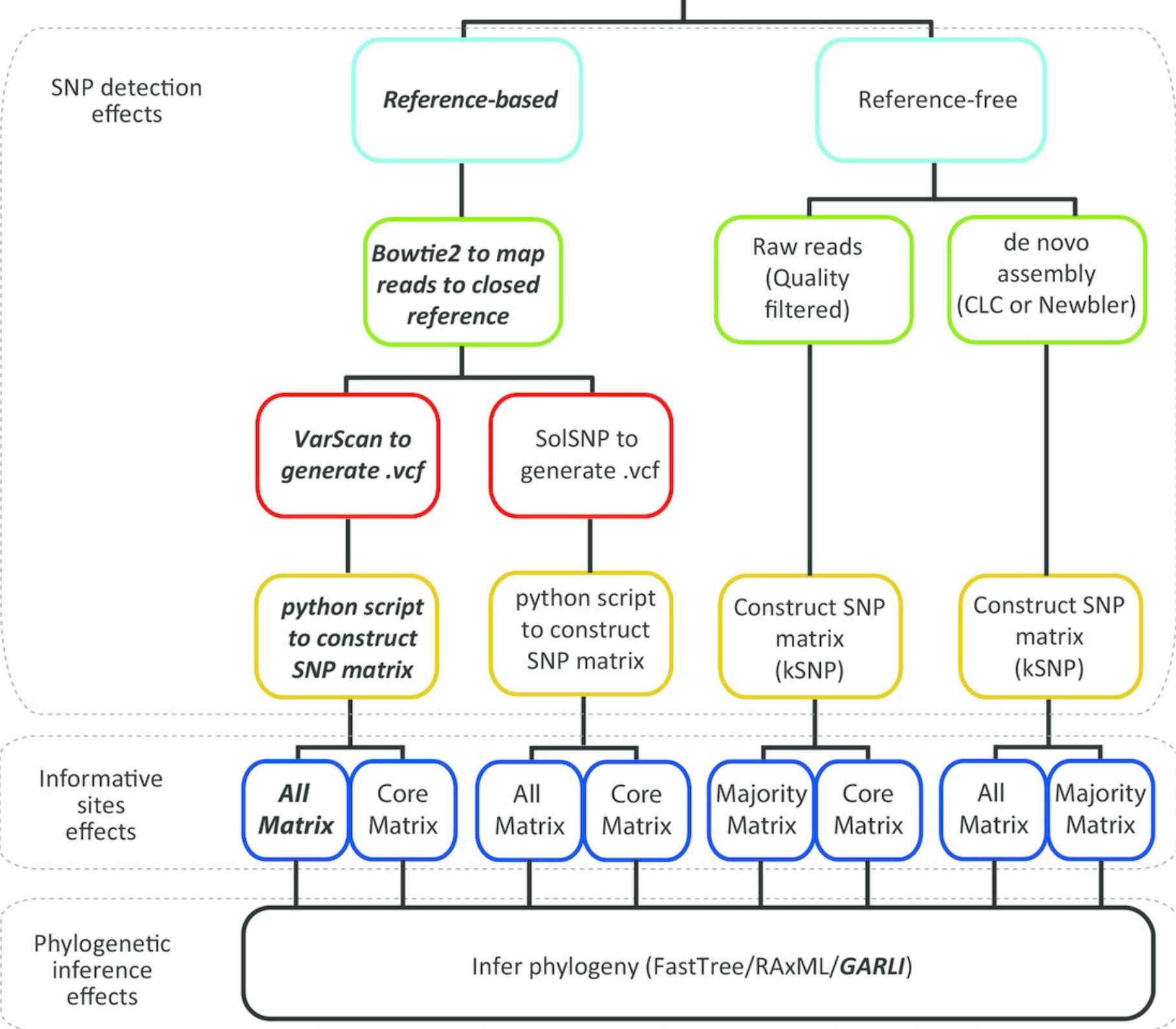




\section{Figure 2}

Figure 2. Characteristics of sample reads by platform.

(a) Average number of reads produced, (b) percent of reads mapped to the reference sample, (c) the number of contigs within the assembly, (d) total number of basepairs assembled; dashed red line represent the bps in the reference, and (e) the N50 of the assembly. Boxes depict the interquartile (IQR) range and whiskers indicate 1.5 IQR; the horizontal black line represents the mean. The lonTorrent platform is abbreviated as IonT. Points represent observed values; points were also offset from one another ('jittered') to reduce overlap. See Table 1 for sample sizes per NGS platform. 

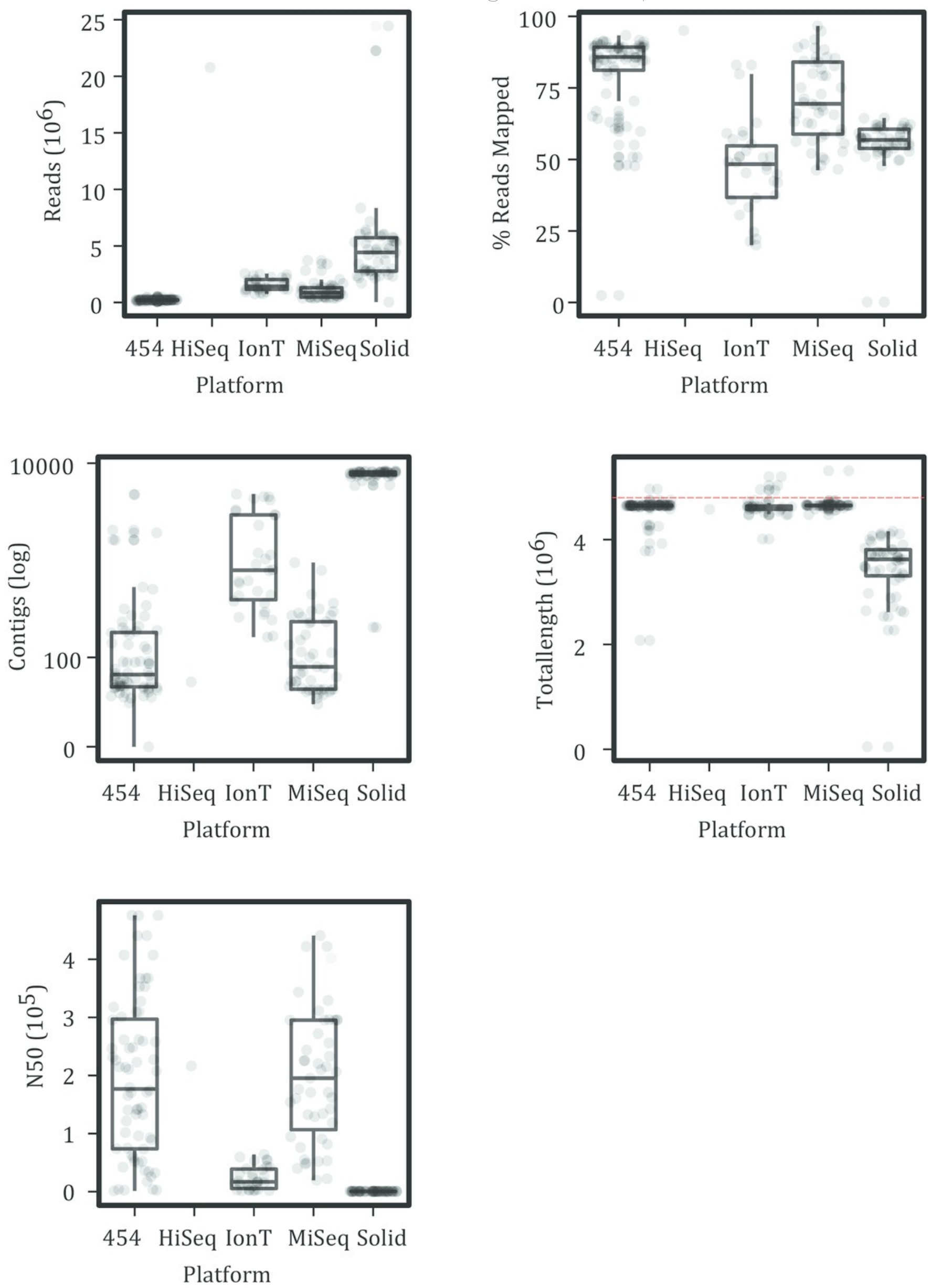

PeerJ reviewing PDF | (v2014:04:1996:2:0:NEW 19 Sep 2014) 


\section{Figure 3}

Figure 3. Proportional number of SNP differences between replicate runs of the same strain on the same platform across the different matrices investigated.

Boxes depict the interquartile (IQR) range and whiskers indicate 1.5 IQR; the horizontal black line represents the mean. 

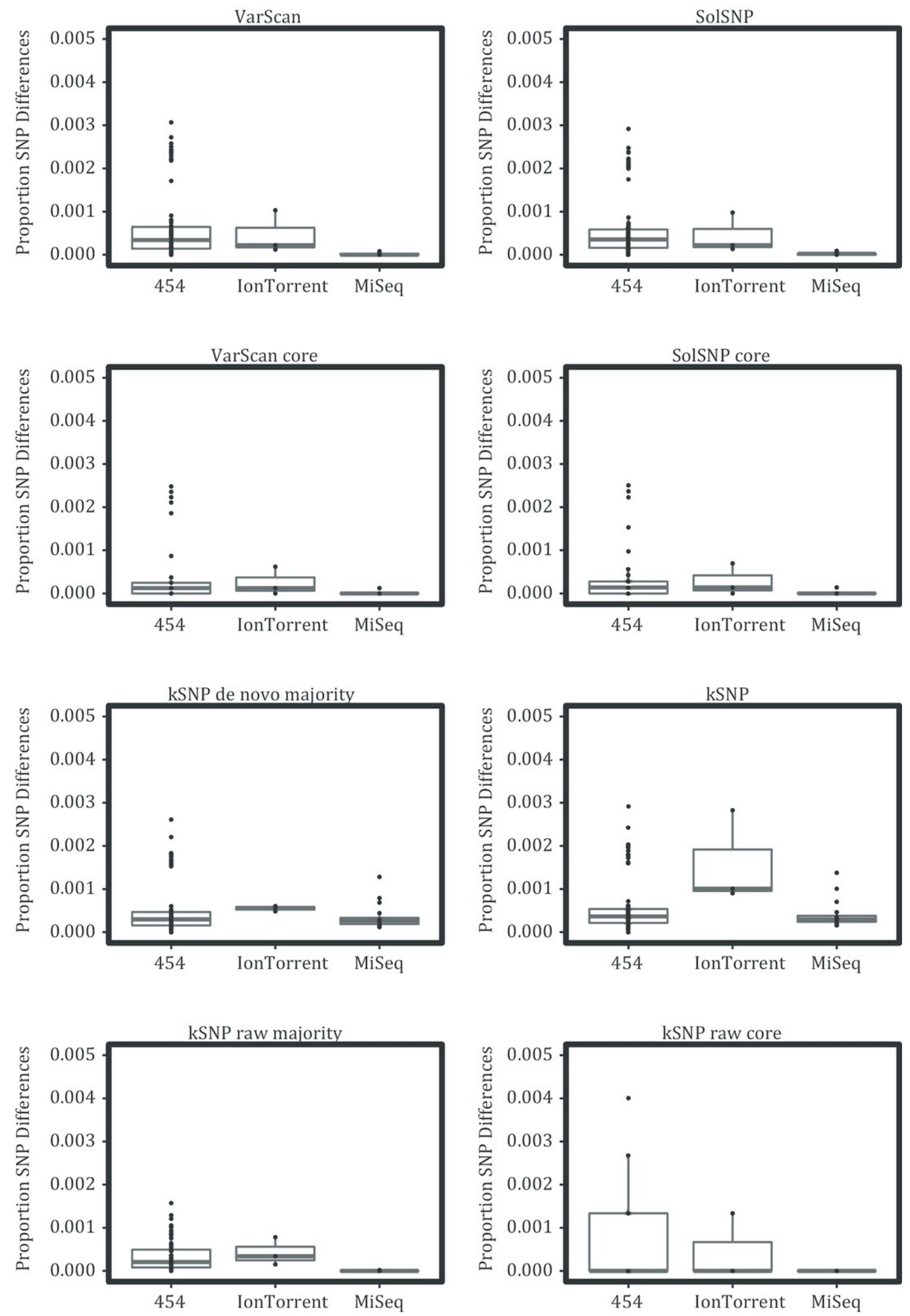


\section{Figure 4}

Figure 4. Boxplots of gsi values for the different topologies inferred and different groupings.

Boxes depict the interquartile (IQR) range and whiskers indicate 1.5 IQR; the horizontal black line represents the mean. Points represent observed values; points were also offset from one another ('jittered') to reduce overlap. See Table S2 for the number of representatives under each grouping. 
Stains Replicated on Same NGS Platforms

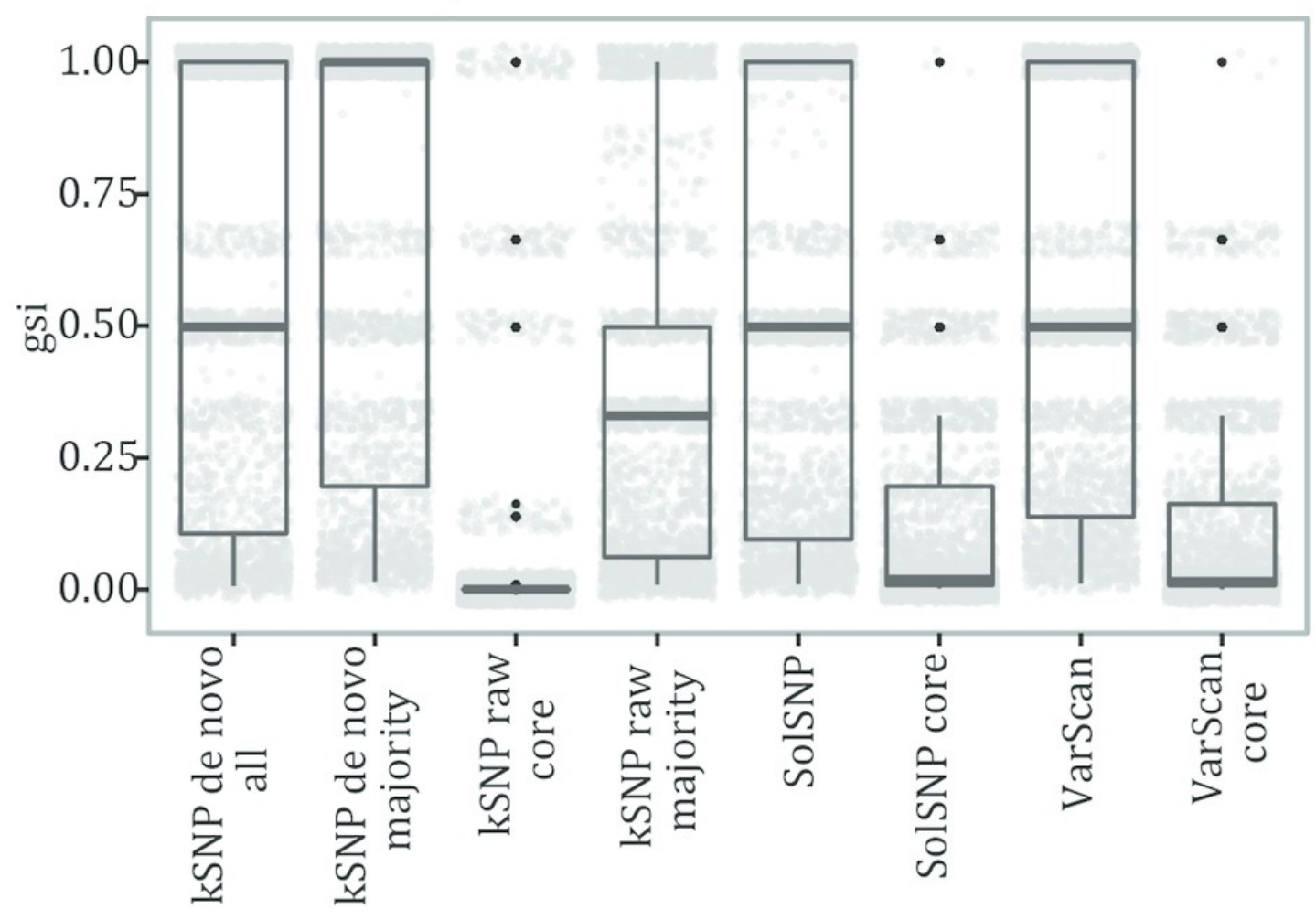

Stains Replicated on Same and Different NGS Platforms

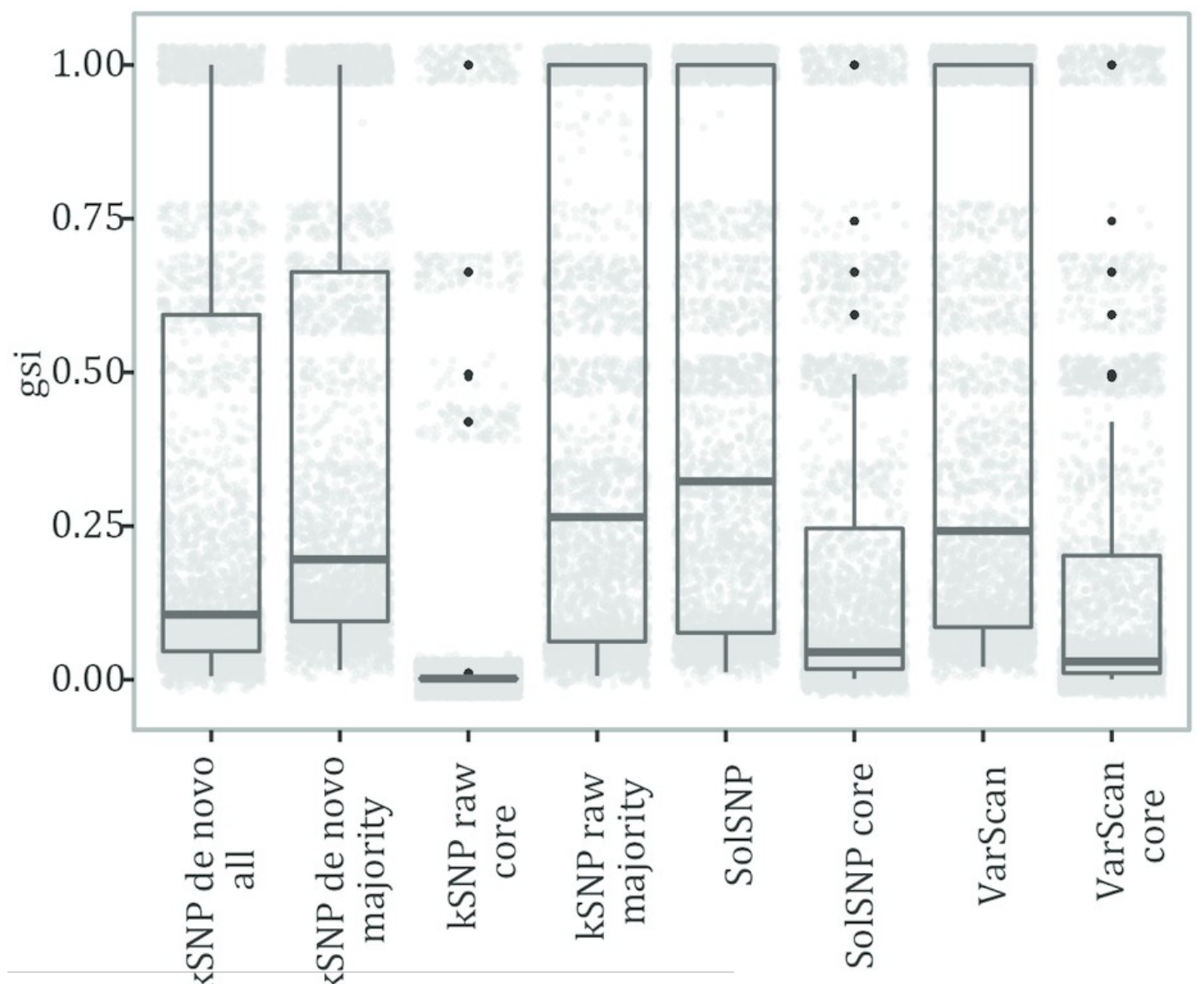




\section{Figure 5}

Figure 5. Violin plots of changes in the symmetric differences statistics across SNP matrices.

Figures show the kernel density estimate of the symmetric differences, broken down by three factors (1. phylogenetic inference package, 2. type of comparison, and 3. type of matrix). Individual observations are omitted due to the large number of them. 


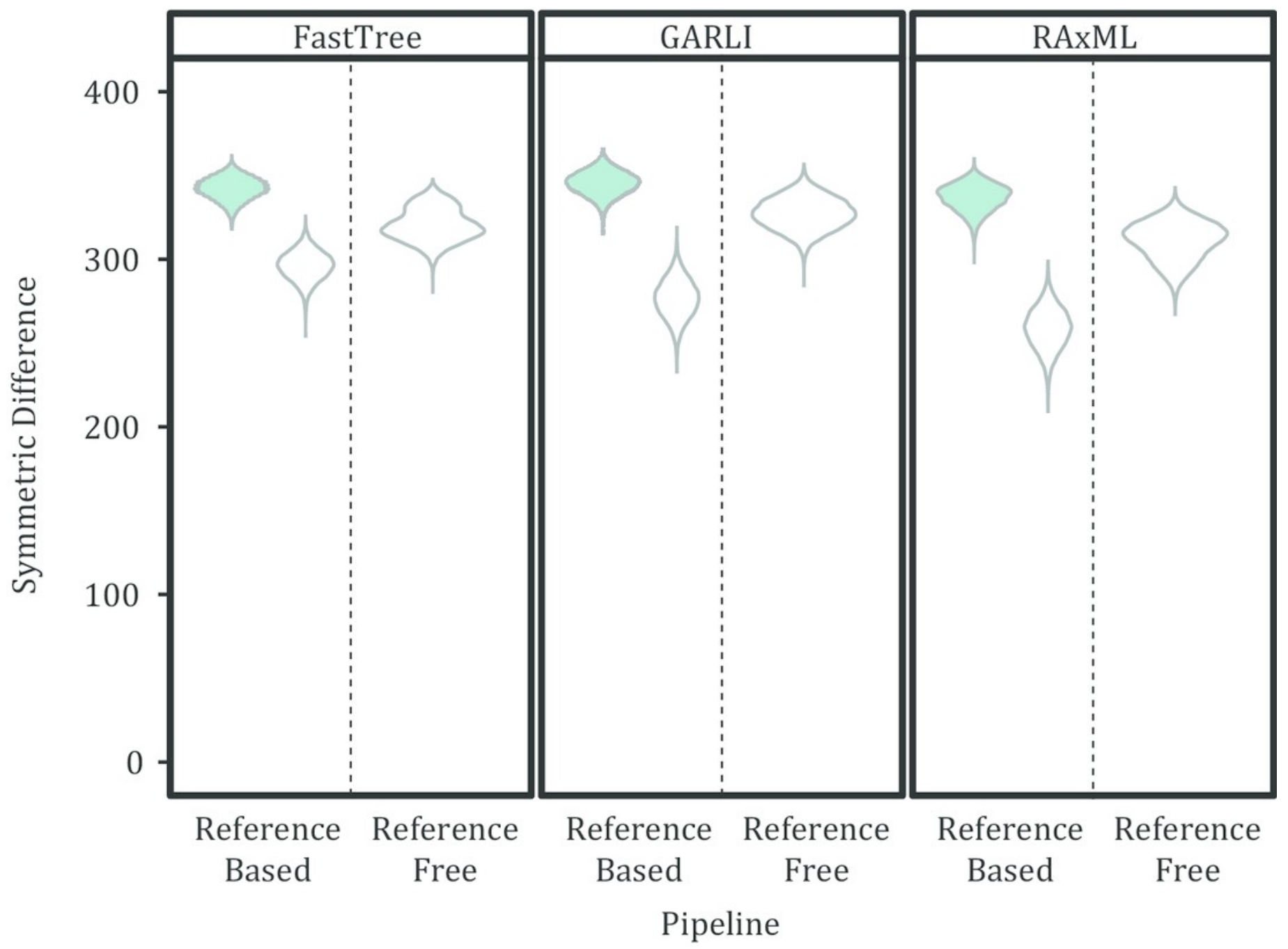

Core Matrix $\square$ Non-Core Matrix 


\section{Figure 6}

Figure 6. Phylograms inferred with RAxML under each of the different matrices created either using a reference-free or reference-based approach.

Red circles represent boostrap support values greater than 0.85 . 


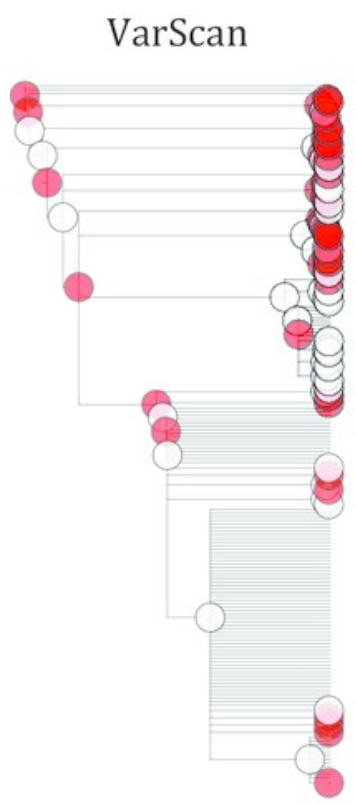

SolSNP

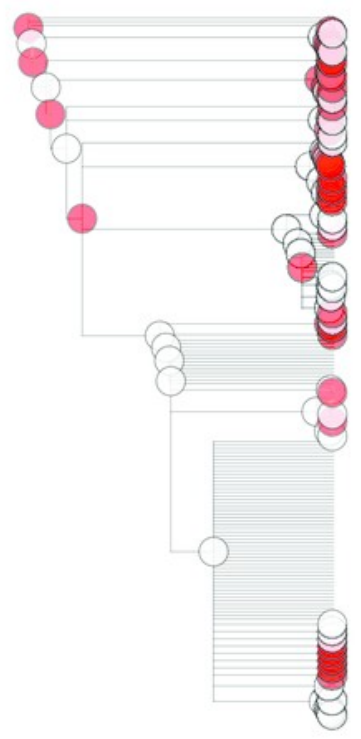

kSNP de novo all
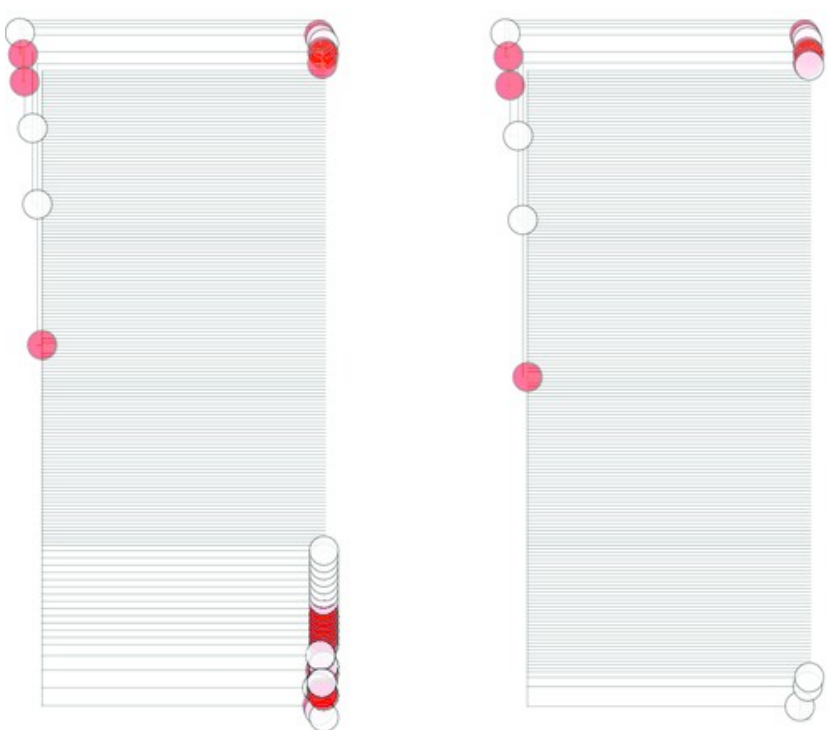

\section{VarScan core}

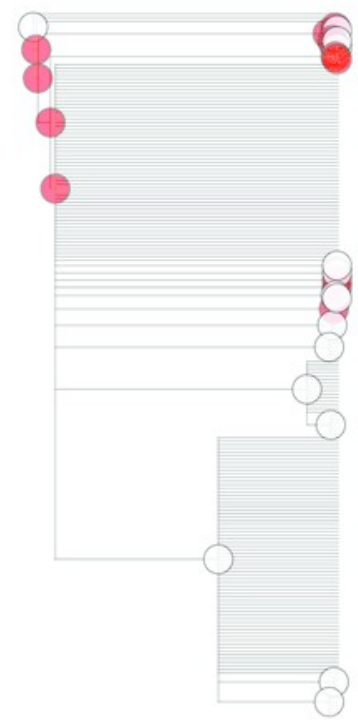

kSNP de novo majority

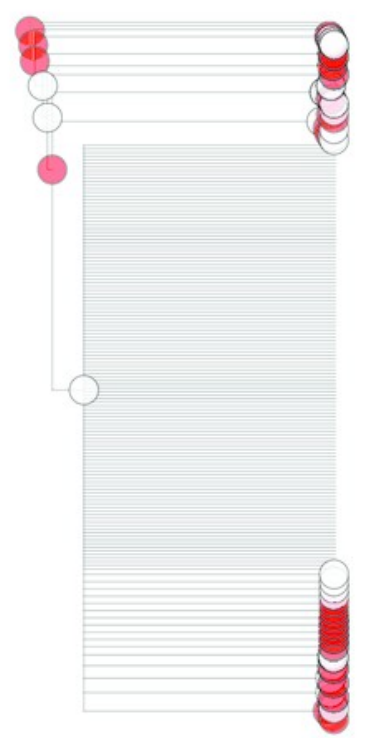

Bootstrap $>85$

Bootstrap $\leq 85$
SolSNP core

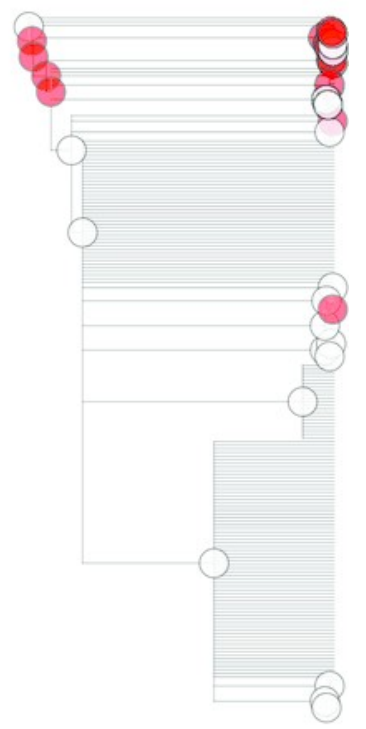

kSNP raw majority

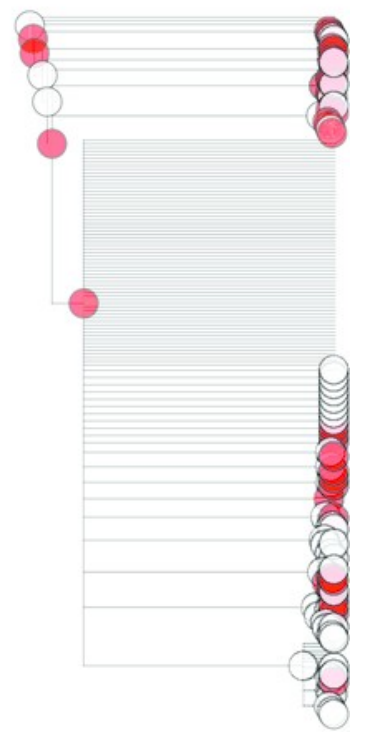




\section{Figure 7}

Figure 7. Violin plots illustrating the differences among inference programs.

Figures show the kernel density estimate of the symmetric differences and topologies are based on the same matrix but different inference methods for both the full 194 sample dataset and for the pared down Allard dataset. 

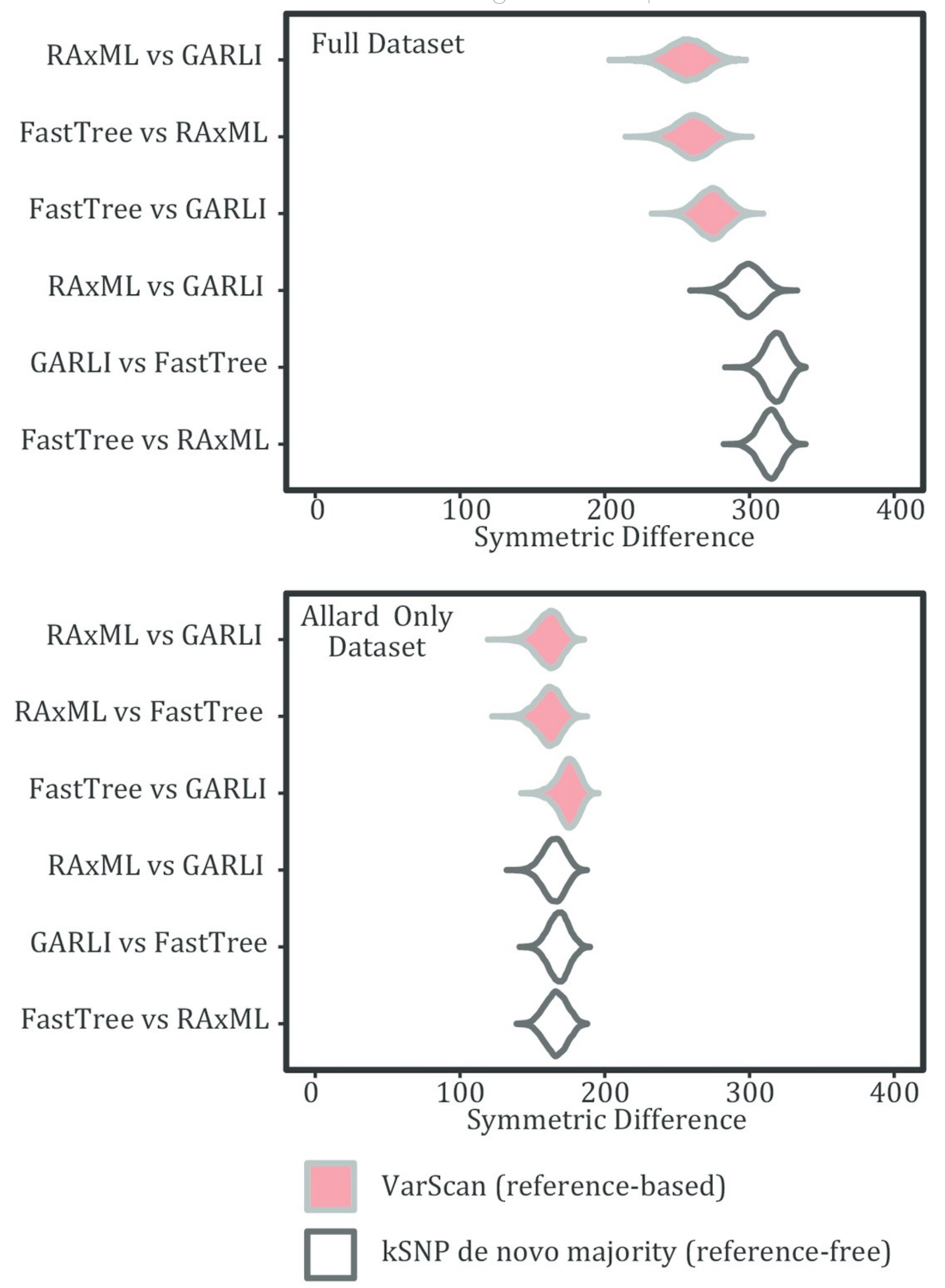


\section{Figure 8}

Figure 8. Topologies inferred with RAXML on matrices that had been pruned to only include strains present in Allard et al. (2012) .

Tips are color-coded based on whether the strain was called as part of the outbreak (red circles) or not part of the outbreak (gray circles). 

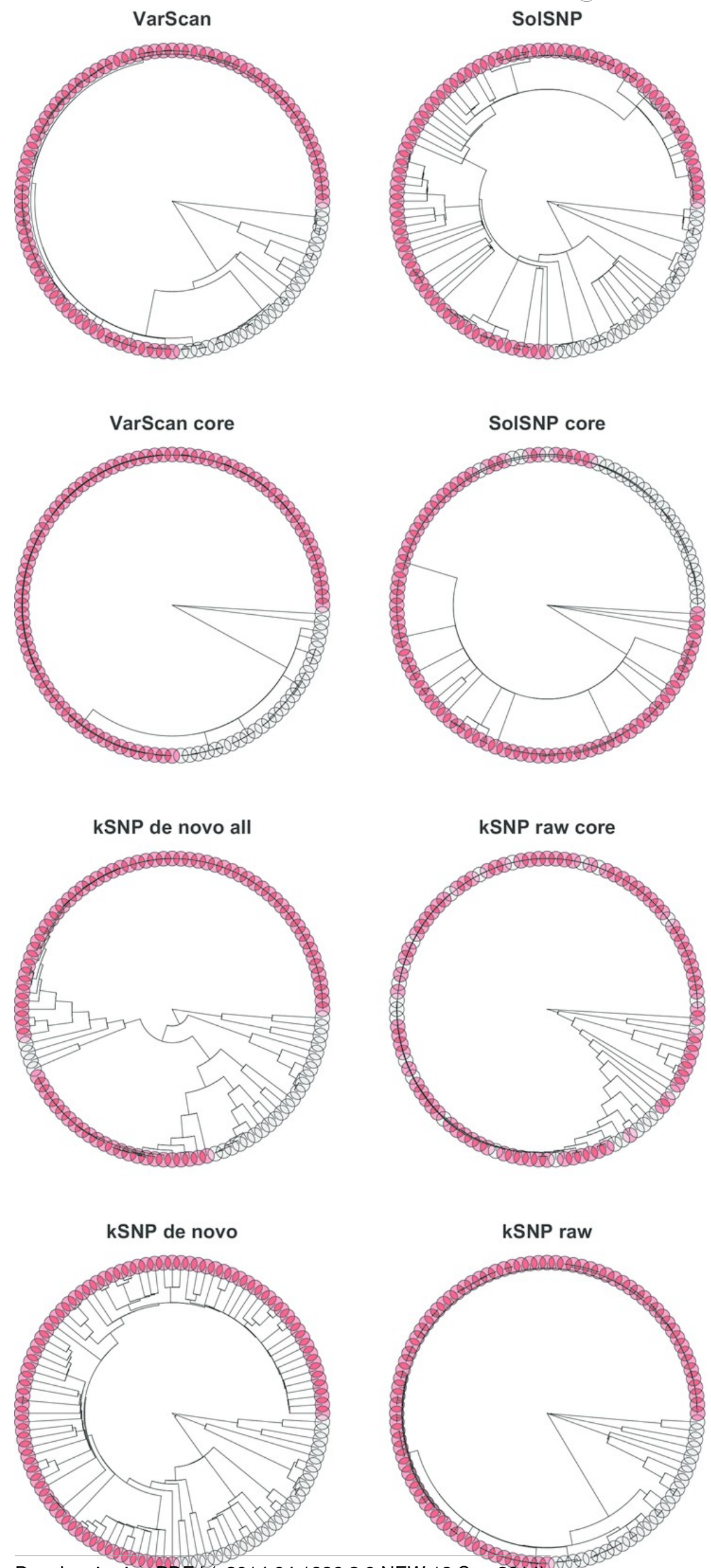

PeerJ reviewing PDF I (v2014:04:1996:2:0:NEW 19 Sep 2014) 


\section{Table 1 (on next page)}

Table 1. Distribution of samples by platform.

The number of samples run on both platforms; diagonal elements contain the number of sequencing runs per platform. 


\begin{tabular}{cccccc}
\hline & 454 & HiSeq & IonTorrent & MiSeq & Solid \\
\hline 454 & 68 & 0 & 17 & 17 & 0 \\
HiSeq & & 1 & 0 & 0 & 0 \\
IonTorrent & & & 30 & 24 & 6 \\
MiSeq & & & & 47 & 5 \\
Solid & & & & & 48 \\
\hline
\end{tabular}




\section{Table 2 (on next page)}

Table 2. SNP Matrix Characteristics.

Summary of the differences across the SNP detection methods by matrix (Core, All, $50 \%$ ) in terms of the number of SNPs, percent missing data, and number of identical sequences. na $=$ not applicable as the core matrices have no missing data; * Not analyzed; ${ }^{2}$ Number of identical sequences within the matrix. 


\begin{tabular}{|c|c|c|c|c|c|}
\hline $\begin{array}{c}\text { SNP } \\
\text { detection }\end{array}$ & Method & Matrix & $\begin{array}{l}\text { Number } \\
\text { of SNPs }\end{array}$ & Missing & $N_{\text {identical }} \#$ \\
\hline \multirow{4}{*}{$\begin{array}{l}\text { Referenc } \\
\text { e-based }\end{array}$} & \multirow{2}{*}{ VarScan } & Core & 8056 & na & 114 \\
\hline & & All & 49307 & $0.7 \%$ & 1 \\
\hline & \multirow{2}{*}{ SoISNP } & Core & 7179 & na & 123 \\
\hline & & All & 45388 & $0.8 \%$ & 2 \\
\hline \multirow{6}{*}{$\begin{array}{l}\text { Referenc } \\
\text { e-free }\end{array}$} & \multirow{3}{*}{$\begin{array}{l}\text { kSNP } \\
\text { de novo }\end{array}$} & Core* & 0 & na & $n / a$ \\
\hline & & $50 \%$ & 51261 & $11 \%$ & 0 \\
\hline & & All & 73236 & $37 \%$ & 0 \\
\hline & \multirow{3}{*}{$\begin{array}{l}\text { kSNP } \\
\text { raw }\end{array}$} & Core & 749 & na & 135 \\
\hline & & $50 \%$ & 54157 & $11 \%$ & 1 \\
\hline & & All* & 2990475 & $96 \%$ & 0 \\
\hline
\end{tabular}




\section{Table 3 (on next page)}

Table 3. Bootstrap support values under each phylogentic inference method and SNP matrix for the bifurcation differentiating outbreak from non-outbreak samples in the Allard et al. (2012) dataset.

na $=$ not applicable as the outbreak samples were not monophyletic. 


\begin{tabular}{cccc}
\hline Matrix & RAxML & GARLI & FastTree \\
\hline VarScan & 93 & 99 & na \\
VarScan core & na & na & 55 \\
SolSNP & 98 & 97 & na \\
SolSNP core & na & na & na \\
kSNP de novo & 91 & 81 & 83 \\
kSNP de novo all & na & na & 65 \\
kSNP raw & 100 & 94 & na \\
kSNP raw core & na & na & na \\
\hline
\end{tabular}

